10 years ESJ

Special edition

\title{
Implementación de Estrategias de Marketing en Tiendas de Abarrotes para Hacer Frente al COVID-19 en Guerrero, México
}

\author{
José Luis Susano García \\ Martha Adela Bonilla Gómez \\ Dra. María del Socorro Cabreara Ríos \\ Universidad Autónoma de Guerrero, Mexico
}

Doi: $10.19044 /$ esj.2021.v17n4p213

Submitted: 28 October 2020

Accepted: 09 December 2020

Published: 07 February 2021
Copyright 2021 Author(s)

Under Creative Commons BY-NC-ND

4.0 OPEN ACCESS

Cite As:

Susano García L.J., Bonilla Gómez A.M. \& Cabreara Ríos M. S.(2021). Implementación de Estrategias de Marketing en Tiendas de Abarrotes para Hacer Frente al COVID-19 en Guerrero, México. European Scientific Journal, ESJ, 17(4), 213.

https://doi.org/10.19044/esj.2021.v17n4p213

\section{Resumen}

En México existen unidades económicas importantes de impacto local que se encargan de comercializar y proveer a manera de pequeñas tiendas, algunos artículos y productos indispensables para el consumo y servicios en los hogares mexicanos principalmente, estas son las conocidas "Tiendas de Abarrotes" o "Tienditas de la Esquina", mismas que tienen un arraigo cultural, pues no solo se encuentran en las poblaciones, barrios y colonias, también sobre existen en grandes ciudades.

Derivado de la pandemia del COVID-19, en México hizo que muchos de los negocios, en particular las MiPYMES se enfrentaron a grades retos, en algunos casos muchos de ellos cerraron de manera temporal y otros más con grandes afectaciones como es el quiebre del negocio.

La investigación se centra en identificar las áreas de oportunidad y la propuesta de estrategias de marketing para las tiendas de abarrotes en 2 poblaciones con 5 mil habitantes y 3 colonias de la ciudad de Chilpancingo, mediante una encuesta aplicada a los dueños por home office, considerando y a través del mystery shopper, las variables consideradas fuero: plaza, comunicación, relaciones públicas y promoción de ventas. 
Los resultados son significativos, porque se identificaron algunas ventajas competitivas que las tiendas de abarrotes tienen comparación con los supermercados o tiendas de conveniencias, por otra parte, permitió mejorar el nivel de posicionamiento de dichas tiendas a nivel local y con ello la fidelización del cliente.

Palabras clave: Mercadotecnia, estrategias, tienda de abarrotes, consumidores

\title{
Implementation of Marketing Strategies in Grocery Stores to Deal with COVID-19
}

\author{
José Luis Susano García \\ Martha Adela Bonilla Gómez \\ Dra. María del Socorro Cabreara Ríos \\ Universidad Autónoma de Guerrero, Mexico
}

\begin{abstract}
In Mexico, there are important economic units that have a local impact and are in charge of marketing and supplying in the form of small stores. These essential items and products are mainly for consumption and services in Mexican homes, and they are well-known "Grocery Stores" or "Tienditas de la Esquina" which have cultural roots. They are not only found in towns and neighborhoods but also exist in large cities. The COVID-19 pandemic in Mexico sprout a lot of challenges for businesses, in particular the MSMEs. In some cases, many of these businesses closed down temporarily while others experienced failure. This paper focuses on identifying the areas of opportunity and proposing marketing strategies for grocery stores in 2 towns with 5,000 inhabitants and 3 neighborhoods in the city of Chilpancingo. This was achieved through a survey distributed to the owners and to home office, considering the mystery shopper. The variables considered jurisdiction: place, communication, public relations, and sales promotion. The results show a significant difference because grocery stores have some competitive advantages when compared to supermarkets or convenience stores. On the other hand, it helps to improve the level of positioning of these stores at the local level and thus customer loyalty.
\end{abstract}

Keywords: Marketing, strategies, grocery store, consumers 


\section{Introduction}

Las consecuencias que ha traído consigo la pandemia ocasionado por el COVID-19, ha generado estragos importantes en diversos sectores, como son la salud, las cuestiones sociales, la educación, la tecnología, el turismo, la producción y la economía, por mencionar algunos.

Las tiendas de abarrotes en México tienen un arraigo histórico y cultura, que son parte de un eslabón económico dentro de la cadena de valor, ello debido a que distribuyen los productos y en ocasiones algunos servicios a lo largo y ancho del país.

Dichas unidades de negocios tienen origen en la época de la conquista española, el concepto de la palabra "abarrotes" comenzó a utilizarse en las colonias españolas de América, donde los establecimientos de venta quedaban "abarrotados" de víveres y otros productos tras la llegada de un buque al puerto procedente de España o de otras colonias (Campos, 2013).

Ello significa que existe una relación entre tienda de abarrotes y los consumidores, mismo que se expresa a un arraigo cultural, ello debido a la cercanía y ubicación de esta tienda en las colonias, barrios, pueblos, ranchos, etc., por lo que la mayoría de las personas ubican para la adquisición de algunos productos.

Por otra parte, también existe información en las que se indican que las MIPYMES enfrentan diferentes problemas que afectan de una u otra manera su competitividad (ANPEC, 2016). En este sentido, en México uno de los sectores que se ha visto afectado por esta globalización y nuevas tendencias de consumo es el sector del comercio al por menor de abarrotes, también conocido como comercio detallista. Y ahora con la pandemia, este tipo de factor externo pasó a ser un elemento más a la lista de problemas que afectan a las tiendas de abarrotes

Por lo que el trabajo versa sobre la importancia y las acciones que las tiendas de abarrotes en México han realizado, sin embargo, debido a las propias circunstancias de la pandemia el estudio se materializa en un sondeo realizado en 2 poblaciones semiurbanas y 2 colonias de la capital del estado de Guerrero, México, ello debido a que se tiene acceso a la obtención de información.

Como objetivo del trabajo se tiene el identificar las estrategias de mercadotecnia que las tiendas de abarrotes han implementado con la finalidad de poder hacer frete a la pandemia.

Por ello el trabajo se justifica desde la perspectiva de generar información de manera general para los negocios, y muy en concreto para el tipo de negocio de venta al detalle que prolifera en México, ello con la finalidad de brindar herramientas a los microempresarios y con ello se genere la toma de decisiones pertinentes. 


\section{Revisión de literatura}

En México, existen más de 900,000 tienditas de abarrotes de las que dependen cinco millones de mexicanos, las cuales ofrecen crédito de venta a 15 millones de consumidores aproximadamente (ANPEC, 2016). Esto significa que existe una importante derrama económica a nivel local y nacional.

Respecto a lo que mencionan Bocanegra y Vázquez (2003, p. 18), al indicar que en México existe un crecimiento constante en las unidades tradicionales de tipo minorista el cual se debe en gran medida al poco capital que se requiere para su inversión y a la alta tasa de desempleo que se vive actualmente en el país. Sin embargo, también se deben considerar elementos mercadológicos que permitan su permanencia, crecimiento y consolidación, ello con la finalidad de mejorar su nivel de competitividad de los negocios.

Es importante hacer mención sobre la conceptualización y características de las Tiendas de Abarrotes tienen, ello con la finalidad de centrar la información y exponer las aportaciones que se han realizado, en este sentido, de acuerdo a Campos (2013), indica que este tipo de tiendas prolifera en muchos de los países, por lo que existen diversas formas de llamar a este tipo de tienda detallista, de acuerdo a Hernández von y Martínez (2016) en otros países se les conoce como tiendas de abarrotes, tiendas de barrio, tiendas de esquina, pulperías, tiendas de ultramarinos, bodegas; dependiendo del país en el que se ubiquen.

Lo anterior se sustenta con lo que Tovar y Mendoza (2009, pág. 18) al mencionar que estos espacios de Tiendas de Abarrotes no solo es un sitio donde se compran y venden productos, es el lugar donde se discute la problemática del barrio y la del país. Las tiendas no van a desaparecer en mucho tiempo, por una simple razón: productos como la leche, el pan, los refrescos y las hortalizas o las frutas, los colombianos jamás dejarán de comprarlas donde el vecino (...)

Por otra parte, los comercios de abarrotes en México, llamados también "changarros" y argumenta que representan el giro comercial más importante en México por la cantidad de unidades de negocio (el 33\% del comercio minorista en México) y su papel en la subsistencia de muchas familias mexicanas (Vargas, 2011, págs. 8-25).

Reyes y Ulibarri (2014), propone una definición la cual expresa que son:

"empresas que se ocupan de la compraventa de diversos productos tales como alimentos enlatados o envasados, jugos y néctares, bebidas gaseosas, artículos de limpieza, lácteos, dulces y frituras, carnes frías, vinos y licores, entre otros; al por menor y en la mayoría de los casos al público en general. Con 0 a 10 empleados, menos de 50 Mts 2 de instalación y no venden bebidas alcohólicas" 
Dichas unidades de negocios se encuentran dentro de la clasificación de micros o pequeñas empresas, ello de acuerdo a la clasificación que hace el INEGI (2018), la las cuales se estratifican de la siguiente manera:

Tabla 1. Estratificación de las empresas por sector económico

\begin{tabular}{|l|l|l|l|}
\hline \multirow{2}{*}{$\begin{array}{l}\text { Tamaño de } \\
\text { Empresa }\end{array}$} & \multicolumn{3}{|l|}{ Número de personas ocupadas } \\
\cline { 2 - 4 } & Manufactura & Comercio & Servicio \\
\hline Micro & $1-10$ & $1-10$ & $1-10$ \\
\hline Pequeña & $11-50$ & $11-30$ & $11-50$ \\
\hline Mediana & $51-250$ & $51-100$ & $21-100$ \\
\hline
\end{tabular}

Fuente: INEGI (2018)

Bajo este contexto, las tiendas de abarrotes son en su mayoría microempresas, es decir, dentro de su operación cuentan entre 0 a 10 empleados de acuerdo a la caracterización del INEGI.

Asimismo, en México en 2018, se registraron poco más de 4.1 millones de micro, pequeñas y medianas empresas (MiPYMES) en México, clasificadas en los sectores de manufacturas, comercio y servicios privados no financieros.

Tabla 2. Número de empresas por tamaño, 2018

\begin{tabular}{|l|l|l|}
\hline \multirow{2}{*}{ Tamaño } & \multicolumn{2}{|l|}{ Empresas } \\
\cline { 3 - 4 } & Número & Participación (\%) \\
\hline Micro & 4057719 & 97.3 \\
\hline $\begin{array}{l}\text { Pequeñas y medianas } \\
\text { empresas }\end{array}$ & 111958 & 2.7 \\
\hline Total & & $\mathbf{4 1 6 9 6 7 7}$ \\
\hline
\end{tabular}

Fuente: INEGI: (2018)

Con relación al comportamiento de distribución y personal ocupado en las MiPYMES en México, se expresa en la siguiente tabla.

Tabla 3. Distribución y personal ocupado en las microempresas de México

\begin{tabular}{|l|l|l|}
\hline SECTOR & DISTRIBUCIÓN & $\begin{array}{l}\text { PERSONAL } \\
\text { OCUPADO }\end{array}$ \\
\hline Comercio & $51 \%$ & $41 \%$ \\
\hline Servicios & $36 \%$ & $38 \%$ \\
\hline Manufacturero & $13 \%$ & $21 \%$ \\
\hline
\end{tabular}

Fuente: INEGI (2018)

Uno de los giros principales de estas empresas es el sector de venta al detalle, esto debido a que el riesgo es menor que en otros giros por la facilidad de entrar o salir de este sector (Anzola, 2010).

Uno de las características respecto a los dueños de estos negocios, es el que presenta FUNDES (2009), al mencionar que el perfil del abarrotero en México posee gran diversidad por múltiples motivos y razones por las cuales 
las personas emprenden un negocio en el comercio tradicional, siendo algunos de ellos, las fuente principal de empleo e ingreso.

Entre las características que tienen las tiendas de abarrotes en la categoría de plaza Hernández von, May y Martínez (2016) mencionan que es un uso común tener un patrocinador de fachada, proveedores importantes como Coca Cola patrocinan cajas de luz para letreros o el rotulado con pintura a cambio de tener su marca como imagen central en el negocio, y lo describen en tres aspectos:

- Mobiliario y equipamiento: se toma en consideración este punto en referencia a la infraestructura bajo la cual se ofrecen los productos o servicios a los clientes. Se observaron el mobiliario y equipamiento como: estantes, vitrinas, refrigerador, aire acondicionado, televisión, equipo de sonido, computadora y sistema de cobro.

- Fachada y letrero: En las tiendas de abarrotes lo básico del merchandising es tener una fachada atractiva, lo cual podría significar contar con un frente bien decorado, con un escaparate atractivo, permanentemente limpio, y con una entrada que facilite y estimule el ingreso. El letrero en la fachada es una referencia importante para los compradores, ya que les permite identificar la mayor o menor formalidad o informalidad de los negocios, en lo que se relaciona a su seriedad y al servicio esperado, esto se observó en el estudio de (May, Martínez, \& Aguilera, 2013).

- Exhibición y surtido: se refiere a la forma en que el local exhibe sus productos, esto contempla: limpieza del local, el acomodo, surtido y variedad de los productos. Contar con una exhibición atractiva de los productos dentro del establecimiento procurando que la cantidad de productos exhibidos sea la adecuada

Existen algunos datos que hablan sobre las problemáticas de las MiPIMES y en especial de las tiendas de abarrotes, pero también hay aportaciones de investigaciones en las que se mencionan que dichos negocios sobreviven aun así con la competencia, caso concreto es el que menciona Suárez (2012) al sugerir que existe un aumento en la probabilidad de sobrevivencia en tiendas de abarrotes cuando llega a una zona un nuevo supermercado de gran tamaño, ello a través de la sinergia empresarial.

Hernández, et al. (2019) mencionan que, respecto a los factores de globalización, en el caso de México uno de los sectores que se ha visto afectado por esta globalización y nuevas tendencias de consumo es el sector del comercio al por menor de abarrotes, también conocido como comercio detallista.

Rodríguez (2010) afirma que las microempresas presentan ciertas desventajas, como las restricciones para adquirir recursos adicionales en el 
negocio, la falta de experiencia del propietario y el tiempo de dedicación en la empresa.

Respecto a la dinámica sobre las problemáticas en materia de competencia se indica información sobre el comportamiento de las tiendas de abarrotes en México de acuerdo a la proliferación de tiendas más grandes como son los supermercados, en este sentido, Bocanegra (2009), hace mención que en el norte del país donde hay mayor ingreso per cápita de la población, las empresas pequeñas y grandes se disputan con mayor intensidad a los compradores.

En este sentido, quienes llevan la delantera son las grandes cadenas de supermercados en sus formatos de hipermercados y megamercados, en claro detrimento de los establecimientos de abarrotes y misceláneas. Por otra parte con respecto al sector detallista, el sur no presenta un gran número de plazas comerciales, hipermercados, supermercados e incluso tiendas de conveniencia comparándola con otras partes la ciudad. Hernández, et al. (2019).

Por otra, respecto a las consecuencias de la pandemia en el ámbito de los negocios es evidente su consecuencia, en razón de ello el BID (2020) menciona que los efectos de la pandemia por la propagación del COVID-19 están sacudiendo las estructuras socioeconómicas a nivel global. Los impactos se estiman en cifras astronómicas de pérdidas de vidas y empleos. Los países de América Latina y el Caribe (ALC) están desplegando medidas sanitarias y económicas para frenar el avance de la epidemia con gran incertidumbre aún sobre la duración del periodo de emergencia sanitaria y las consecuencias reales de la pandemia sobre la población y el tejido productivo.

Datos significativos con relación a las afectaciones en los negocios de cualquier tamaño y giro se han estado evidenciado mediante informes, reportes, estudios y papers que dan a conocer la realidad que vive la economía, por ejemplo CEPYME (2020) destaca que el 96\% de estas empresas afirman que se están viendo afectadas negativa o muy negativamente ante esta situación y un $67 \%$ considera que las expectativas generales en 2020 para su negocio son malas o muy malas.

En caso concreto de México, de acuerdo al INEGI (2020), menciona que el $93.4 \%$ son microempresas, 5.9\% Pequeñas y Medianas y el $0.7 \%$ grandes empresas, del total de las microempresas, el 59.6\% instrumentó paros técnicos y temporales, por más de 21 días, mientras que el $40.4 \%$ de las microempresas no lo hizo.

Por otra parte del total de las empresas en México, el 93.2\% fue afectada de una u otra manera, entre las variables de afectación destacan:

- Disminución de los ingresos

- Baja de demanda 
- Escasez de insumos y/o productos

- Reducción del personal

- Reducción de remuneraciones y/o prestaciones

En la actualidad los empresarios mexicanos derivado del COVID-19 se encuentran en la disyuntiva de cómo hacer frente a esta pandemia y con ello mitigar de una mejor manera las consecuencias negativas que esta pandemia ha traído, existen algunos documentos, guías, protocolos que sirven de apoyo para dar herramientas y sugerencias a los microempresarios en materia de prevención (OIT, 2020; BID, 2020; CANACO-SERVITUR, 2020; CANIRAC, 2020).

\section{Método}

Para la realización de la investigación, de tipo mixta con la finalidad de tener versiones cuantitativas y cualitativas sobre los dos contextos en los que se ubican las tiendas de abarrotes (semi urbano y urbano) y concreto sobre las estrategias de mercadotecnia y con ello identificar si existes diferencias.

Se consideraron las tiendas de abarrotes en estos lugares debido a que se tiene acceso a información de ellas, además de que se cuenta con personas cercanas (familiares, conocidos y alumnos de Gestión Empresarial) a los investigadores que colaboraron, dicha colaboración fue sin costo alguno, asimismo se les brindó una capacitación en materia de mystery shopper y home office.

Tabla 4. Universo total de las tiendas de abarrotes

\begin{tabular}{|l|l|l|}
\hline \multirow{2}{*}{ MUNICIPIO } & LUGAR & $\begin{array}{l}\text { NÚMERO DE TIENDAS } \\
\text { DE ABARROTES }\end{array}$ \\
\hline San Marcos & Población 1 & 85 \\
\hline Tecoanapa & Población 2 & 115 \\
\hline \multirow{3}{*}{ Chilpancingo } & Colonia 1 & 19 \\
\cline { 2 - 3 } & Colonia 2 & 17 \\
\cline { 2 - 3 } & Colonia 3 & 13 \\
\hline \multicolumn{2}{|l|}{ TOTAL DE TIENDAS DE ABARROTES } & $\mathbf{2 8 6}$ \\
\hline
\end{tabular}

Posterior de conocer el universo total por tipo de población, se procedió a determinar el número de muestra por población y colonia, ello con la finalidad de tener una muestra representativa del número de tiendas de abarrotes. 
Tabla 5. Muestra por tienda de abarrotes

\begin{tabular}{|l|l|l|}
\hline MUNICIPIO & LUGAR & MUESTRA \\
\hline San Marcos & Población 1 & 70 \\
\hline Tecoanapa & Población 2 & 89 \\
\hline \multirow{4}{*}{ Chilpancingo } & Colonia 1 & 19 \\
\cline { 2 - 3 } & Colonia 2 & 17 \\
\cline { 2 - 3 } & Colonia 3 & 13 \\
\hline TOTAL DE TIENDAS DE ABARROTES & $\mathbf{2 4 0}$ \\
\hline
\end{tabular}

Para la recolección de datos, se contó con la colaboración de 68 personas, las cuales se encargaron de realizar las observaciones y entrevistas correspondientes, para ello se cuidó las cuestiones de salud a través de la sana distancia y la dotación de materiales como caretas y gel antibacterial.

Se realizó un proceso de capacitación en materia de conocimiento del concepto de mystery shopper; como una figura del que se encarga de recogida de datos que utiliza la observación para calificar una serie de variables, así como la técnica de entrevistas por vía telefónica (CATI), implementadas a través de trabajo en casa (home office). La capacitación se llevó a cabo por gropos de lugares (población,1, población 2, colonia,1, colonia 1, colonia 2, colonia 3), se capacitó del 27 al 31 de julio de 2020. Para realizar la actividad del comprador misterioso, esta se llevó a cabo los meses de agosto y septiembre dónde se realizaron compras y la verificación de las estrategias que mercadotecnia que se consideraron en la investigación.

\section{Hallazgos}

Respecto a los resultados, es importante mencionar que para el paper se presentan los hallazgos que se obtuvieron de la actividad del mystery shopper, ello con la finalidad de no saturar al lector con la información debido a que los datos son numerosos y se pueden utilizar para otro documento.

La Plaza como primer variable considerada seis indicadores que son: Limpieza del local, la sana distancia, la utilización de gel, sanitizante o tapete, uso de acrílico, uso de cubreboca del dueño o vendedor, y colocación de reja maya o cinta protectora de no acceso (Ver Tabla 6). 
Tabla 6. Variable plaza y sus indicadores

\begin{tabular}{|l|l|l|l|l|l|l|l|l|l|l|l|}
\hline \multirow{2}{*}{ Indicador } & \multicolumn{3}{|l|}{ Población 1 } & \multicolumn{2}{l|}{ Población 2 } & \multicolumn{2}{l|}{ Colonia 1 } & \multicolumn{2}{l|}{ Colonia 2 } & \multicolumn{2}{l|}{ Colonia 3 } \\
\cline { 2 - 13 } & Sí & No & Sí & No & Sí & No & Sí & No & Sí & No \\
\hline Limpieza del local & $95 \%$ & $5 \%$ & $95 \%$ & $5 \%$ & $100 \%$ & 0 & $100 \%$ & 0 & $84 \%$ & $6 \%$ \\
\hline Sana distancia & $91 \%$ & $9 \%$ & $96 \%$ & $4 \%$ & $89 \%$ & $11 \%$ & $88 \%$ & $12 \%$ & $85 \%$ & $5 \%$ \\
\hline $\begin{array}{l}\text { Utilización de gel, } \\
\text { sanitizante o tapete }\end{array}$ & $100 \%$ & 0 & $100 \%$ & 0 & $100 \%$ & 0 & $100 \%$ & 0 & $100 \%$ & 0 \\
\hline Uso de acrílico & $82 \%$ & $18 \%$ & $93 \%$ & $7 \%$ & $89 \%$ & $11 \%$ & $88 \%$ & $12 \%$ & $77 \%$ & $13 \%$ \\
\hline $\begin{array}{l}\text { Uso de cubreboca } \\
\text { del dueño o o } \\
\text { vendedor }\end{array}$ & $100 \%$ & 0 & $100 \%$ & 0 & $100 \%$ & 0 & $100 \%$ & 0 & $100 \%$ & 0 \\
\hline $\begin{array}{l}\text { Colocación de reja, } \\
\text { maya o cinta } \\
\text { protectora de no } \\
\text { acceso }\end{array}$ & $100 \%$ & 0 & $100 \%$ & 0 & $100 \%$ & 0 & $100 \%$ & 0 & $100 \%$ & 0 \\
\hline
\end{tabular}

Los resultados son significativos al identificar que no existe diferencia entre las tiendas de abarrotes tanto de las ciudades como de las poblaciones semi urbanas, pues el total de ella realizó las 6 estrategias consideradas en la investigación, destaca también el hallazgo que los dueños de las unidades de negocios están conscientes de la importancia de adaptar considerar las recomendaciones por parte de los organismos de salud, comercio y gobierno tanto local, estatal como nacional.

Estos resultados identifican la importancia que los dueños de las tiendas de abarrotes han tomado antes esta pandemia, ello por una parte porque son fuentes de abastecimiento de su zona de influencia, como fuente de generación de ingresos y empleos.

Destaca que el $100 \%$ de las tiendas de abarrotes realiza la utilización de gel, sanitizante o tapete y colocación de reja maya o cinta protectora de no acceso.

Comunicación es la segunda variable, la cual contiene seis indicadores: Letrero de uso de cubrebocas, letrero de servicios de envío, letrero de sana distancia, letrero de información del COVID-19 y letrero de información de hospitales para atención médica (Ver Tabla 7). 
Tabla 7. Variable comunicación y sus indicadores

\begin{tabular}{|l|l|l|l|l|l|l|l|l|l|l|l|}
\hline \multirow{2}{*}{ Indicador } & \multicolumn{2}{|l|}{ Población 1 } & \multicolumn{2}{l|}{ Población 2 } & \multicolumn{2}{l|}{ Colonia 1 } & \multicolumn{2}{l|}{ Colonia 2 } & \multicolumn{2}{l|}{ Colonia 3 } \\
\cline { 2 - 13 } & Sí & No & Sí & No & Sí & No & Sí & No & Sí & No \\
\hline $\begin{array}{l}\text { Letrero de uso } \\
\text { de cubrebocas }\end{array}$ & $100 \%$ & 0 & $100 \%$ & 0 & $100 \%$ & 0 & $100 \%$ & 0 & $100 \%$ & 0 \\
\hline $\begin{array}{l}\text { Letrero de } \\
\text { servicios de } \\
\text { envío }\end{array}$ & $\begin{array}{l}60 \\
(85 \%)\end{array}$ & $\begin{array}{l}10 \\
(15 \%)\end{array}$ & $\begin{array}{l}85 \\
(95 \%)\end{array}$ & $\begin{array}{l}4 \\
(5 \%)\end{array}$ & 0 & 0 & 0 & 0 & 0 & 0 \\
\hline $\begin{array}{l}\text { Letrero de sana } \\
\text { distancia }\end{array}$ & $100 \%$ & 0 & $100 \%$ & 0 & $100 \%$ & 0 & $100 \%$ & 0 & $100 \%$ & 0 \\
\hline $\begin{array}{l}\text { Letrero de } \\
\text { información del } \\
\text { COVID-19 de }\end{array}$ & $82 \%$ & $58 \%$ & $60 \%$ & $40 \%$ & $57 \%$ & $43 \%$ & $76 \%$ & $24 \%$ & $61 \%$ & $39 \%$ \\
\hline $\begin{array}{l}\text { Letrero } \\
\text { información de de } \\
\text { hospitales para } \\
\text { atención médica }\end{array}$ & $30 \%$ & $70 \%$ & $29 \%$ & $69 \%$ & $37 \%$ & $63 \%$ & $29 \%$ & $71 \%$ & $30 \%$ & $70 \%$ \\
\hline
\end{tabular}

Fuente: Elaboración propia de acuerdo a los resultados obtenidos

La comunicación es un elemento importante para que se generar información del negocio a los consumidores y una retroalimentación de la misma, es este sentido es importante mencionar que el $100 \%$ de las tiendas de abarrotes comunican el uso de cubrebocas y guardar la sana distancia.

Un dato significativo, destaca que en su mayoría no se expone un letrero de información del COVID-19 y letrero de información de hospitales para atención médica, aunque no es obligación de las tiendas de abarrotes realizarlos, se considera importante para generan confianza por parte de los consumidores.

En el caso de información de servicios de envíos destaca el caso de las tiendas de abarrotes de las poblaciones consideran esta estrategia, mientras que en el caso de las tiendas de las 3 colonias no realizan el servicio de envíos.

La tercera variable es la Promoción de ventas, la cual se integra por tres indicadores: Facilidades de pagos (fiado), pagos seccionados y descuentos (Ver Tabla 8).

Tabla 8. Variable Promoción de ventas y sus indicadores

\begin{tabular}{|l|l|l|l|l|l|l|l|l|l|l|l|}
\hline \multirow{2}{*}{ Indicador } & \multicolumn{2}{|l|}{ Población 1 } & \multicolumn{2}{l|}{ Población 2 } & \multicolumn{2}{l|}{ Colonia 1 } & \multicolumn{2}{l|}{ Colonia 2 } & \multicolumn{2}{l|}{ Colonia 3 } \\
\cline { 2 - 11 } & Sí & No & Sí & No & Sí & No & Sí & No & Sí & No \\
\hline $\begin{array}{l}\text { Facilidades de pagos } \\
\text { (fiado) }\end{array}$ & $100 \%$ & 0 & $100 \%$ & 0 & $100 \%$ & 0 & $100 \%$ & 0 & $100 \%$ & 0 \\
\hline Pagos seccionados & $100 \%$ & 0 & $100 \%$ & 0 & $100 \%$ & 0 & $100 \%$ & 0 & $100 \%$ & 0 \\
\hline Descuentos & $80 \%$ & $20 \%$ & $78 \%$ & $22 \%$ & $79 \%$ & $21 \%$ & $70 \%$ & $30 \%$ & $77 \%$ & $33 \%$ \\
\hline
\end{tabular}

Fuente: Elaboración propia de acuerdo a los resultados obtenidos 
A pesar de que son indicadores que debido al tipo de micronegocio se pueden considerar como no favorables o benéficos debido a que sus ingresos se generan de la venta de los productos, destaca la información que el 100\% de las tiendas de abarrotes si han considerado las facilidades de pagos (fiado) y pagos seccionados. Es importante destacar que para estos dos indicadores los dueños de los micronegocios realizan las facilidades de pago por dos motivos, el primero a cantidades razonables de pago y la segunda a personas que conocen de años atrás a las que tiene mayor confianza y en ocasiones para quienes son clientes poco frecuentes les piden dejar un porcentaje del costo total.

Finalmente, en materia de descuentos se realiza a ciertos tipos de productos y el descuento oscila entre un 10 y 15 por ciento.

Este indicador permite a las tiendas de abarrotes poder competir en el mercado y generar ventajas competitivas en comparación de las tiendas departamentales, supermercados y de conveniencia.

La última variable corresponde a las estrategias de Relaciones Públicas, las cuales están compuestas por 6 indicadores que son: Venta de productos de vecinos (salsa, pan, tortillas, frijoles cocidos, verduras, frutas secas, etc.), empatía por parte del dueño o vendedor, identificación de la confianza para otorgar créditos por parte del dueño o vendedor, saludos y despedida por parte del dueño o vendedor, apoyo en especie a familias que lo requieren y venta de productos fuera de horarios (emergencia) (Ver Tabla 9). Tabla 9. Variable Relaciones Públicas y sus indicadores

\begin{tabular}{|c|c|c|c|c|c|c|c|c|c|c|}
\hline \multirow{2}{*}{ Indicador } & \multicolumn{2}{|c|}{\begin{tabular}{|l|} 
Población 1 \\
\end{tabular}} & \multicolumn{2}{|c|}{ Población 2} & \multicolumn{2}{|c|}{ Colonia 1} & \multicolumn{2}{|c|}{ Colonia 2} & \multicolumn{2}{|c|}{ Colonia 3} \\
\hline & Sí & No & Sí & No & Sí & No & Sí & No & Sí & No \\
\hline $\begin{array}{l}\text { Venta de productos de } \\
\text { vecinos (salsa, pan, } \\
\text { tortillas, frijoles } \\
\text { cocidos, verduras, } \\
\text { frutas secas, etc.) }\end{array}$ & $100 \%$ & 0 & $100 \%$ & 0 & $100 \%$ & 0 & $100 \%$ & 0 & $100 \%$ & 0 \\
\hline $\begin{array}{l}\text { Empatía por parte del } \\
\text { dueño o vendedor }\end{array}$ & $94 \%$ & $6 \%$ & $89 \%$ & $11 \%$ & $78 \%$ & $22 \%$ & $70 \%$ & $30 \%$ & $77 \%$ & 33 \\
\hline $\begin{array}{l}\text { Identificación de la } \\
\text { confianza para otorgar } \\
\text { créditos por parte del } \\
\text { dueño o vendedor }\end{array}$ & $\begin{array}{l}66 \\
(94 \%)\end{array}$ & $\begin{array}{l}4 \\
(6 \%)\end{array}$ & $\begin{array}{l}80 \\
(89 \%)\end{array}$ & $\begin{array}{l}9 \\
(11 \%)\end{array}$ & $\begin{array}{l}15 \\
(78 \%)\end{array}$ & $\begin{array}{l}4 \\
(22 \%)\end{array}$ & $\begin{array}{l}12 \\
(70 \%)\end{array}$ & $\begin{array}{l}5 \\
(30 \%)\end{array}$ & $\begin{array}{l}10 \\
(77 \%)\end{array}$ & $\begin{array}{l}3 \\
(33 \%)\end{array}$ \\
\hline $\begin{array}{l}\text { Saludos y despedida } \\
\text { por parte del dueño o } \\
\text { vendedor }\end{array}$ & $100 \%$ & 0 & $100 \%$ & 0 & $100 \%$ & 0 & $100 \%$ & 0 & $100 \%$ & 0 \\
\hline $\begin{array}{lll}\text { Apoyo en } & \text { especie } & \text { a } \\
\text { familias } & \text { que } & \text { lo } \\
\text { requieren } & & \\
\end{array}$ & 50 & 20 & 70 & 19 & 11 & 8 & 11 & 6 & 8 & 5 \\
\hline 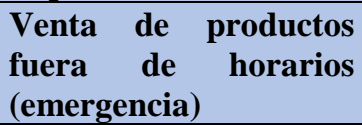 & $100 \%$ & 0 & $100 \%$ & 0 & $100 \%$ & 0 & $100 \%$ & 0 & $100 \%$ & 0 \\
\hline
\end{tabular}

Fuente: Elaboración propia de acuerdo a los resultados obtenidos 
De las estrategias de Relaciones Públicas destacan tres indicadores que el $100 \%$ de los dueños de las tiendas de abarrotes realiza la venta de productos de vecinos (salsa, pan, tortillas, frijoles cocidos, verduras, frutas secas, etc.), ello con la finalidad de apoyar a la economía local realizando acciones de mercadotecnia solidaria.

De igual manera el $100 \%$ realizan un saludo y despedida, ello identifica la educación y respecto hacia los consumidores por parte de los dueños o vendedores. Y de igual manera el total de los dueños o vendedores realizan ventas fuera de horarios esta acción es con el fin de apoyar en situaciones de salud o emergencia de higiénica para bebés.

Empatía por parte del dueño o vendedor y la identificación de la confianza para otorgar créditos por parte del dueño o vendedor es un indicador que obtuvieron el mismo porcentaje, ello puede ser debido a que para la generación de confianza y empatía es necesario que pase un tiempo de trato y comunicación entre los dueños-vendedores y consumidores, sin embargo la tendencia es en aumento.

\section{Discusión}

El interés del trabajo se justifica a razón de ser una necesidad para los investigadores conocer la situación que están viviendo día a días los microempresarios de las tiendas de abarrotes y con ello generar propuestas de mercadotecnia en beneficio de la comunidad empresarial.

Otro aspecto tiene que ver con la poca información de investigación empírica en materia de tiendas de abarrotes y en especial las ubicadas en poblaciones semiurbanas y rurales, generando con ello la pertinencia del estudio.

En líneas anteriores se indica que las tiendas de abarrotes son parte de la cultura mexicana, en razón de ello se demuestra que existe dicha relación entre la tienda y la relación que se genera con los consumidores (confianza).

Es importante destacar que es necesario aumentar el numero de universo con la finalidad de hacer un estudio más representativo y en diversos estados de México.

\section{Conclusiones}

En la actualidad existen diferentes situaciones o problemáticas que afectan de manera directa e indirecta a las MiPYMES y en particular a las tiendas de abarrotes, por mencionar factores externos están, la inseguridad, la situación social, la economía, los aspectos tecnológicos, la competencia, las tendencias de consumo y ahora en la actualidad, las cuestiones de salud, como el COVID-19.

Entre los problemas más a los que se han enfrentado las tiendas de abarrotes son las pérdidas en materia de ventas y descomposición de 
productos, respecto a las mermas en productos estos se reflejan en la reducción de un cierto número de mercancías, la no actualización de un stock, por otra parte, se han identificado aspectos como el cierre temporal de algunas tiendas de abarrotes.

La mercadotecnia es una alternativa para la solución de algunos de los problemas, para ello se deben desarrollar estrategias que permitan aumentar las ventas a través de mediante estrategias de comercialización creativas.

Se deben establecer canales de comercialización directos como la entrega de productos a domicilio aprovechando la cercanía entre las tiendas de abarrotes y los hogares. El uso de las redes sociales es una oportunidad para dar a conocer y comunicar a los consumidores los productos y ofertas para con ello aumentar las ventas.

Finalmente, el comercio electrónico es otra herramienta importante a destacar ya que con ello se facilita la transacción monetaria y se evita de la manipulación de monedas y papel moneda, evitando así un posible contagio.

Las tiendas de abarrotes no desaparecerán, pues son parte de la historia y la cultura empresarial mexicana con presencia en todas las poblaciones del país localidades, comunidades, pueblos, pequeñas ciudades y grandes ciudades, por lo que es importante que se adapten a los diversos cambios que se generan.

La creatividad y la innovación son dos elementos mercadológicos necesarios para que las microempresas desarrollen con la finalidad de que puedan afrontar las problemáticas que se presenten, tengan mayor participación en el mercado y un mejor nivel de competitividad.

\section{References:}

1. ANPEC (2016). Cómo salvar de la muerte a tu tiendita. Revista Entrepreneur. Recuperado de: https://www.entrepreneur.com/article/279000

2. Anzola, S. (2010). Administración de Pequeñas Empresas. México: Mc Graw Hill.

3. BID. Banco Interamericano de Desarrollo (2020). Respuestas al COVID-19 desde la ciencia, la innovación y el desarrollo productivo. Documento. Recuperado de: https://publications.iadb.org/es/respuestas-al-covid-19-desde-laciencia-la-innovacion-y-el-desarrollo-productivo

4. Bocanegra, C. (2009). Una mirada al comercio minorista del norte de México. Revista Universidad de Sonora. Recuperado de: http://www.revistauniversidad.uson.mx/indice.php?id=26 
5. Bocanegra, C \& Vázquez, M. (2003). Modernización en el comercio detallista. Revista de Información y Análisis, 2 (23)., INEGI, México, pp. $17-25$

6. Campos, S. (2013). Tienda de abarrotes. Semiperiodismo. Recuperado de: https://semperiodismo.wordpress.com/2013/01/17/tienda-deabarrotes/

7. CANACO-SERVITUR (2020). Protocolo para el regreso de los comercios, los servicios y el turismo a la actividad económica durante la emergencia sanitaria del COVID-19. Recuperado de: https://www.concanaco.com.mx/protocolo-para-el-regreso-de-loscomercios-los-servicios-y-el-turismo-a-la-actividad-economicadurante-la-emergencia-sanitaria-covid-19/\#1

8. CANIRAC (2020). Protocolo de prevención COVID-19. Mesa segura. Recuperado de:

https://canirac.org.mx/images/notas/files/Protocolo\%20Reapertura\%2 0-\%20Mesa\%20Segura_compressed.pdf?fbclid=IwAR3e0vLPZ1A1ifWCCTF9mdPsHwAwYepRrOVZp9XX5x3W6FJNc81ELqJzjE

9. CEPYME (2020). Pymes y COVID-19: hacia una recuperación sostenible. Recuperado de: https://www.pactomundial.org/wpcontent/uploads/2020/06/Gu\%C3\%ADa-Pymes-y-COVID-19_hacia-una-recuperaci\%C3\%B3n-sostenible.pdf

10. FUNDES (2009). Programa de Comercio Detallista. Serie Nuestra Experiencia, No.3. México D.F., México.

11. Hernández, et al. (2019). Calidad en el servicio en tiendas de abarrotes. Una perspectiva de género. Revista del Centro de Graduados e Investigación. Instituto Tecnológico de Mérida. Volumen 34. Pág. 99-107. Recuperado de http://www.revistadelcentrodegraduados.com/p/blog-page_1.html

12. Hernández Von, May \& Martínez (2016). Factores comerciales relacionados a la supervivencia de las tiendas de abarrotes tradicionales, Caso Región 101, Cancún, Quintana Roo, México. IBFR, Revista Global de Negocios Vol. 4, No. 8, 2016, pp. 61-70. Recurado de: https://www.theibfr.com/download/rgn/2016-rgn/rgnv4n8-2016/RGN-V4N8-2016-6.pdf

13. INEGI (2018). Resultados de la Encuesta Nacional sobre Productividad y Competitividad de las Micro, Pequeñas y Medianas Empresas (ENAPROCE). Comunicado de prensa núm. 448/19. Recuperado de: https://www.inegi.org.mx/contenidos/saladeprensa/boletines/2019/es peciales/ENAPROCE2018.pdf 
14. (2020). Encuesta sobre el Impacto Económico Generado por COVID-19 en las Empresas. Recuperado de:

https://www.inegi.org.mx/contenidos/programas/ecovidie/2020/doc/p resentacion_ECOVIDIE.pdf

15. May, F., Martínez, M. G., \& Aguilera, O. (2013). Análisis del comercio informal en la Reg. 101, Benito Juárez, Quintana RooMéxico. Un estudio Longitudinal. IBFR, Revista Global de Negocios. San José Costa Rica.

16. OIT. Organización Internacional del Trabajo (2020). Prevención y mitigación de COVID-19 en el trabajo para Pequeñas y Medianas Empresas. Lista de Comprobación y Manual de Comprobación. Recuperado de: https://www.ilo.org/wcmsp5/groups/public/--ed_dialogue/---lab_admin/documents/publication/wcms_753623.pdf

17. Reyes E.E., \& Ulibarri, H. (2014). Estudio comparativo de la calidad en el servicio de las tiendas de abarrotes y una cadena de minisúpers del sur de la ciudad de Mérida, Yucatán". Instituto Tecnológico de Mérida. Recuperado de: https://prezi.com/co7vccbn6fnz/copy-oftiendas-de-abarrotes/

18. Rodríguez Valencia, J. (2010). Administración de pequeñas y medianas empresas. México: Cengage Learning.

19. Suárez, P. (2012). Abre tu tienda de abarrotes. Soy Entrepreneur. Recuperado de: http://www.soyentrepreneur.com/9533-tu-tienda-deabarrotes.html

20. Tovar, S. A., \& Mendoza, C. X. (2009). La importancia de la tienda de barrio como canal de distribución aplicado en la localidad de Candelaria. Bogotá: Universidad del Rosario.

21. Vargas, O. (2011). El micro y pequeño comercio de abarrotes en México. México, D.F. Tesina UNAM, Facultad de Economía. 